Article

\title{
Is the Perceived Fruit Accessibility Related to Fruit Intakes and Prevalence of Overweight in Disadvantaged Youth: A Cross-Sectional Study
}

\author{
Narae Yang $(1)$ and Kirang Kim *(1) \\ Department of Food Science and Nutrition, College of Natural Sciences, Dankook University, Dandae-ro, \\ Dongnam-gu, Cheonan-si, Chungnam 31116, Korea; skfo2581@daum.net \\ * Correspondence: kirangkim@dankook.ac.kr; Tel.: +82-41-529-6373
}

Received: 23 September 2020; Accepted: 19 October 2020; Published: 29 October 2020

\begin{abstract}
Background: Few investigations have studied the relationship between home and school food environments, fruit intakes, and prevalence of overweight in children and adolescents from disadvantaged backgrounds. This study aimed to determine whether food environments for fruit intake at household and school levels affect fruit intakes and risk of overweight among children and adolescents with low household income. Methods: Students $(n=3148)$ in Seoul, Korea completed questionnaires pertaining to select aspects of their food environments, frequency of fruit intakes, and weight status. Chi-square tests and logistic regressions evaluated associations between the aforementioned variables. Results: Participants consumed fruit an average of 0.77 times per day, though its frequency increased when fruit accessibility was perceived positively. The percentage of overweight participants was $23.5 \%$ for boys and $22.8 \%$ for girls. Generally, fruit intake frequency was linked to a lower prevalence of overweight. Regular provision of fruit in school lunches was associated with a reduced risk of overweight among elementary school girls (odds ratio (OR): 0.52, 95\% confidence interval (CI): 0.30-0.92), and having someone at home to prepare fruit was associated with a reduced risk of overweight in elementary school boys (OR: $0.64,95 \%$ CI: $0.43-0.94$ ) and girls (OR: 0.63, 95\% CI: 0.43-0.93). Conclusions: The frequency of fruit intake was low among disadvantaged youth. Increasing access to fruit in their food environments appears to enhance consumption and lower the risk of overweight, especially for elementary school girls.
\end{abstract}

Keywords: food environment; fruit; children; adolescents; obesity; overweight; home; school

\section{Introduction}

The epidemic of pediatric overweight and obesity has expanded steadily over the past few decades, currently reaching more than 370 million children worldwide [1]. Consequences of early excess adiposity include a heightened risk of psychological problems such as depression, low self-esteem, and disordered eating in the immediate term, as well as cardiovascular disease and various cancers upon adulthood [2-5]. Adult obesity, implemented in child obesity, makes it difficult to lose weight [6]. Therefore, it is important to prevent obesity in childhood [6]. The prevalence of obesity among children and adolescents has increased in Asia [7]. Although the rising trends of body mass index (BMI) have flattened in high-income countries, the prevalence of obesity is still high in worldwide [7].

Obesity has been explained with reduced intakes of healthy foods and unhealthy eating behaviors [8-11]. Especially fruit, which are rich in water and fiber, to enhance satiety, and low in energy density [12], have been known to prevent obesity, coronary heart disease, stroke, cardiovascular disease, total cancer, and all-cause mortality [13-16]. Obesity prevention interventions that take into account healthy food intake, such as fruit, are needed. For effective obesity intervention, it is important 
to identify the relationship between eating healthy foods and obesity and to find the main determinants that affect obesity. Previous intervention methods for eating healthy foods to prevent obesity have focused on individual behavior changes. However, individual level interventions fundamentally could not change the obesogenic environment, which have been reported to have little effect on long-term improvement $[17,18]$. In recent years, the ecological model has been applied to promote health in the public health sector. The multilevel interventions that take an account for both individual and environmental factors have been paid attention for effective behavior change. Among environmental factors, food environments include political, economic, social, physical, and natural environment factors which affect accessibility, availability, and affordability of foods [19,20]. The food environments have been known as an important factor related to food choice and dietary intakes [19,20]. For children and adolescents, the household is the first physical and social environment to learn about food intake [21], and the school is responsible for their lunches and another setting to make them eat healthy food [21]. Thus, for effective intervention, factors at household and school levels that affect the availability and accessibility of foods as well as at the individual level should be included [22].

It has been reported that the food environment has a greater effect on health and nutritional status in the vulnerable group than in the general group [23], but most of the studies have been conducted on the general group [24], and there is a dearth of research exploring how food environments at home and school affect fruit intakes and risk of overweight among disadvantaged children and adolescents. Therefore, research is needed to determine how the food environments of vulnerable children is related to healthy food intake and obesity. For this study, we surveyed low socioeconomic status youth to determine how select aspects of their food environments associate with consumption of fruit and weight status. Therefore, the objective of this study was to investigate whether food environments at household and school levels affect fruit intakes and risk of overweight among disadvantaged children and adolescents.

\section{Methods}

\subsection{Study Population}

Participants were recruited in 2015 from the Community Childcare Center, located in Seoul, Korea. They provide welfare services (e.g., protection, education, and meals) after school to youth from disadvantaged backgrounds (e.g., beneficiaries of national basic livelihood or single-parent families, etc.) [25]. During this time, the Community Childcare Center participated in the Healthy Fruit Basket Program, which aims to prevent the development of chronic diseases by providing access to fresh fruits [25,26]. In total, 4154 students (mean age; elementary school: $10.4 \mathrm{y}$, middle and high school: 14.3 y) were recruited; however, only 3148 were included in the final analyses because they supplied information for all the variables of interest and were of an eligible weight status (i.e., normal and overweight) $[27,28]$. The Institutional Review Board approved this study, and all participants gave written informed consent and assent (DKU 2015-10-016).

\subsection{Weight Status}

Height and weight were measured by a trained measurer at the public health centers or the Community Childcare Centers using a nationally certified weight and height scale. BMI was calculated as weight $(\mathrm{kg})$ divided by height squared $\left(\mathrm{m}^{2}\right)$. According to the 2007 Korean National Growth Charts for children and adolescents [29], classification of normal weight was defined as having a body weight between the fifth and eighty-fourth percentiles, based on one's gender and age; classification of overweight was defined as having a body weight in the eighty-fifth percentile or above, or BMI $25 \mathrm{~kg} / \mathrm{m}^{2}$ or greater, based on one's gender and age. 


\subsection{Fruit Intakes}

Fruit frequency questionnaires examined fruit intakes from the month preceding study enrollment. Scoring ranged from 0 (never) to 9 ( $3+$ times per day). All responses were converted into times per day and collapsed into two categories ( $<0.5$ times per day vs. $\geq 0.5$ times per day) to determine the association with overweight.

\subsection{Food Environments}

Participants were asked five questions about their food environments, adapted from those employed in a previous investigation [30]. Here, three dimensions of the physical environment were evaluated: (1) Availability of fruit at home, (2) accessibility of fruit at home (i.e., is there someone who prepares fruit for children to eat?), and (3) accessibility of fruit at school (i.e., does the school provide fruit twice a week?). For the social environment, questions inquired about the habit of frequently eating fruit among parents and friends. Responses for the questions ranged from 1 (strongly disagree) to 5 (strongly agree), and all were collapsed into categories of disagree (strongly disagree, disagree), neutral, and agree (agree, strongly agree).

\subsection{Statistical Analysis}

Chi-square tests assessed the distribution of participants' general characteristics, the food environment, and weight status according to the frequency of fruit intakes and aspects of the food environment. T-tests evaluated differences in the frequency of fruit intakes by sex and grade level. An analysis of variance and Scheffe's post-hoc test determined differences between the food environments. Logistic regressions analyzed the effects of fruit intakes and food environments on weight status, showing odds ratios (ORs), 95\% confidence intervals (95\% CI). All analyses were performed by SPSS Statistics (v. 23.0); $p \leq 0.05$ was considered statistically significant.

\section{Results}

General characteristics of the participants are shown in Table 1. Overall, $49.0 \%$ were boys and $71.5 \%$ were in elementary school. The percentage of overweight for boys and girls was $23.5 \%$ and $22.8 \%$, respectively. The percentage of overweight for elementary school, and middle and high school students was $24.3 \%$ and $20.4 \%$, respectively. There was a significant difference of weight status between grades, and the percentage of overweight was high in elementary school students.

Table 1. General characteristics of subjects ${ }^{1}$.

\begin{tabular}{lcccc}
\hline & $\begin{array}{c}\text { All } \\
(n=3148)\end{array}$ & $\begin{array}{c}\text { Normal Weight } \\
(\boldsymbol{n}=\mathbf{2 4 1 9 )}\end{array}$ & $\begin{array}{c}\text { Overweight } \\
(\boldsymbol{n}=\mathbf{7 2 9})\end{array}$ & $\boldsymbol{p}^{\mathbf{2}}$ \\
\hline Sex & & & & \\
$\quad$ Boys & $1542(49.0)$ & $1179(76.5)$ & $363(23.5)$ & 0.617 \\
$\quad$ Girls & $1606(51.0)$ & $1240(77.2)$ & $366(22.8)$ & \\
\hline Grade & & & & \\
$\quad$ Elementary school & $2251(71.5)$ & $1705(75.7)$ & $546(24.3)$ & 0.021 \\
$\quad$ Middle and high school & $897(28.5)$ & $714(79.6)$ & $183(20.4)$ & \\
\hline Sex, grade & & & & \\
$\quad$ Boys & $1061(68.8)$ & $797(75.1)$ & $264(24.9)$ & 0.065 \\
$\quad$ Elementary school & $481(31.2)$ & $382(79.4)$ & $99(20.6)$ & \\
$\quad$ Middle and high school & & & & \\
$\quad$ Girls & $1190(74.1)$ & $908(76.3)$ & $282(23.7)$ & 0.142 \\
$\quad$ Elementary school & $416(25.9)$ & $332(79.8)$ & $84(20.2)$ & \\
$\quad$ Middle and high school & $416)$ &
\end{tabular}

${ }^{1}$ Qualitative variables are presented as $\mathrm{n}(\%) .{ }^{2} p$-values for differences between the weight statuses were obtained by a chi-square test. 
Table 2 details aspects of the participants' food environments. Overall, 59.5\% to 63.2\% of participants responded positively (i.e., affirmed the availability of fruit at home, a regular provision of fruit in school lunches, having someone at home to prepare fruit for them, and a family habit of frequently eating fruit). Between the sexes, girls perceived their food environments more positively than boys $(p<0.001)$. Between the grade levels, elementary school students perceived their food environments more positively than middle and high school students $(p<0.001)$.

Table 2. Select aspects of participants' food environments ${ }^{1}$.

\begin{tabular}{|c|c|c|c|c|c|}
\hline & \multirow{2}{*}{ All } & \multicolumn{2}{|c|}{ Sex } & \multicolumn{2}{|r|}{ Grade } \\
\hline & & Boys & Girls & Elementary School & Middle and High School \\
\hline \multicolumn{6}{|c|}{ Availability of fruit at home } \\
\hline Disagree & $458(14.5)$ & $238(15.4)$ & $220(13.7)$ & $313(13.9)$ & $145(16.2)$ \\
\hline Neutral & $816(25.9)$ & $441(28.6)$ & $375(23.3)$ & $557(24.7)$ & $259(28.9)$ \\
\hline Agree & $1874(59.5)$ & $863(56.0)$ & $1011(63.0)$ & $1381(61.4)$ & $493(55.0)$ \\
\hline$p^{2}$ & & \multicolumn{2}{|c|}{$<0.001$} & \multicolumn{2}{|r|}{0.004} \\
\hline \multicolumn{6}{|c|}{ Regular provision of fruit in school lunches } \\
\hline Disagree & $259(8.2)$ & $155(10.1)$ & $104(6.5)$ & $145(6.4)$ & $114(12.7)$ \\
\hline Neutral & $959(30.5)$ & $504(32.7)$ & $455(28.3)$ & $624(27.7)$ & $335(37.3)$ \\
\hline Agree & $1930(61.3)$ & $883(57.3)$ & $1047(65.2)$ & $1482(65.8)$ & $448(49.9)$ \\
\hline$p^{2}$ & & \multicolumn{2}{|c|}{$<0.001$} & \multicolumn{2}{|r|}{$<0.001$} \\
\hline \multicolumn{6}{|c|}{ Having someone at home to prepare fruit } \\
\hline Disagree & $439(13.9)$ & $226(14.7)$ & $213(13.3)$ & $295(13.1)$ & $144(16.1)$ \\
\hline Neutral & $718(22.8)$ & $393(25.5)$ & $325(20.2)$ & $471(20.9)$ & 247 (27.5) \\
\hline Agree & $1991(63.2)$ & $923(59.9)$ & $1068(66.5)$ & $1485(66.0)$ & $506(56.4)$ \\
\hline$p^{2}$ & & \multicolumn{2}{|c|}{$<0.001$} & \multicolumn{2}{|r|}{$<0.001$} \\
\hline \multicolumn{6}{|c|}{ Family habit of eating fruit frequently } \\
\hline Disagree & $282(9.0)$ & $143(9.3)$ & $139(8.7)$ & $193(8.6)$ & $89(9.9)$ \\
\hline Neutral & $954(30.3)$ & $518(33.6)$ & $436(27.1)$ & $618(27.5)$ & $336(37.5)$ \\
\hline Agree & $1912(60.7)$ & $881(57.1)$ & $1031(64.2)$ & $1440(64.0)$ & $472(52.6)$ \\
\hline$p^{2}$ & & \multicolumn{2}{|c|}{$<0.001$} & \multicolumn{2}{|r|}{$<0.001$} \\
\hline \multicolumn{6}{|c|}{ Friends' habit of eating fruit frequently } \\
\hline Disagree & $544(17.3)$ & $299(19.4)$ & $245(15.3)$ & $364(16.2)$ & $180(20.1)$ \\
\hline Neutral & $1378(43.8)$ & $710(46.0)$ & $668(41.6)$ & $936(41.6)$ & $442(49.3)$ \\
\hline Agree & $1226(38.9)$ & $533(34.6)$ & $693(43.2)$ & $951(42.2)$ & $275(30.7)$ \\
\hline$p^{2}$ & & \multicolumn{2}{|c|}{$<0.001$} & \multicolumn{2}{|r|}{$<0.001$} \\
\hline
\end{tabular}

Table 3 shows the relationship between aspects of participants' food environments and frequency of fruit intakes. Regardless of sex or grade level, fruit intakes differed according to how one's food environment was perceived. Here, the group who perceived their food environments positively was found to consume fruit 0.87 to 0.95 times per day, whereas the group who perceived their food environments negatively was found to consume fruit 0.42 to 0.67 times per day $(p<0.001)$. Interestingly, there were no differences in fruit intakes between sexes when they shared the same perception about their food environments. However, when both grade levels responded positively about their food environments, consumption of fruit was found to be higher among elementary school students. For groups displaying a negative or neutral perception, frequency of fruit intakes was comparable. 
Table 3. Relationship between aspects of participants' food environments and frequency of fruit intakes ${ }^{1}$.

\begin{tabular}{|c|c|c|c|c|c|}
\hline & \multirow{2}{*}{ All } & \multicolumn{2}{|c|}{ Sex } & \multicolumn{2}{|r|}{ Grade } \\
\hline & & Boys & Girls & Elementary School & Middle and High School \\
\hline All & $0.77 \pm 0.17$ & $0.74 \pm 0.02^{\mathrm{a}}$ & $0.80 \pm 0.02^{b}$ & $0.85 \pm 0.02^{a}$ & $0.59 \pm 0.02^{b}$ \\
\hline$p^{2}$ & \multicolumn{3}{|c|}{0.036} & & $<0.001$ \\
\hline \multicolumn{6}{|c|}{ Availability of fruit at home } \\
\hline Disagree & $0.47 \pm 0.03^{\mathrm{a}}$ & $0.46 \pm 0.04^{\mathrm{a}}$ & $0.49 \pm 0.04^{\mathrm{a}}$ & $0.51 \pm 0.04^{\mathrm{a}}$ & $0.39 \pm 0.03^{\mathrm{a}}$ \\
\hline Neutral & $0.55 \pm 0.02^{\mathrm{a}}$ & $0.54 \pm 0.03^{\mathrm{a}}$ & $0.55 \pm 0.03^{a}$ & $0.60 \pm 0.03 \mathrm{ab}$ & $0.44 \pm 0.03^{a}$ \\
\hline Agree & $0.95 \pm 0.02^{b}$ & $0.92 \pm 0.03^{b}$ & $0.96 \pm 0.03^{b}$ & $1.03 \pm 0.03^{c}$ & $0.71 \pm 0.03^{b}$ \\
\hline$p^{2}$ & $<0.001$ & \multicolumn{2}{|c|}{$<0.001$} & \multicolumn{2}{|r|}{$<0.001$} \\
\hline \multicolumn{6}{|c|}{ Regular provision of fruit in school lunches } \\
\hline Disagree & $0.65 \pm 0.05^{\mathrm{a}}$ & $0.62 \pm 0.06^{\mathrm{a}}$ & $0.69 \pm 0.08^{a b}$ & $0.64 \pm 0.07$ acd & $0.66 \pm 0.06^{\mathrm{ad}}$ \\
\hline Neutral & $0.62 \pm 0.02^{\mathrm{a}}$ & $0.62 \pm 0.03^{\mathrm{a}}$ & $0.63 \pm 0.03^{\mathrm{a}}$ & $0.70 \pm 0.03^{\mathrm{a}}$ & $0.47 \pm 0.03^{\mathrm{cd}}$ \\
\hline Agree & $0.87 \pm 0.02^{b}$ & $0.83 \pm 0.03^{b}$ & $0.89 \pm 0.03^{b}$ & $0.93 \pm 0.02^{b}$ & $0.64 \pm 0.03 \mathrm{ad}$ \\
\hline$p^{2}$ & $<0.001$ & \multicolumn{2}{|c|}{$<0.001$} & \multicolumn{2}{|r|}{$<0.001$} \\
\hline \multicolumn{6}{|c|}{ Having someone at home to prepare fruit } \\
\hline Disagree & $0.60 \pm 0.04^{\mathrm{a}}$ & $0.55 \pm 0.05^{\mathrm{a}}$ & $0.64 \pm 0.05^{\mathrm{a}}$ & $0.67 \pm 0.05^{\text {ae }}$ & $0.43 \pm 0.03 \mathrm{ad}$ \\
\hline Neutral & $0.53 \pm 0.02^{\mathrm{a}}$ & $0.52 \pm 0.03^{\mathrm{a}}$ & $0.55 \pm 0.04^{\mathrm{a}}$ & $0.58 \pm 0.03^{\mathrm{ac}}$ & $0.44 \pm 0.03^{\mathrm{cd}}$ \\
\hline Agree & $0.90 \pm 0.02^{b}$ & $0.88 \pm 0.03^{b}$ & $0.91 \pm 0.03^{b}$ & $0.97 \pm 0.02^{b}$ & $0.70 \pm 0.03^{\mathrm{e}}$ \\
\hline$p^{2}$ & $<0.001$ & \multicolumn{2}{|c|}{$<0.001$} & \multicolumn{2}{|r|}{$<0.001$} \\
\hline \multicolumn{6}{|c|}{ Family habit of eating fruit frequently } \\
\hline Disagree & $0.42 \pm 0.04^{\mathrm{a}}$ & $0.42 \pm 0.05^{\mathrm{a}}$ & $0.43 \pm 0.05^{\mathrm{a}}$ & $0.46 \pm 0.05^{a b}$ & $0.33 \pm 0.03 \mathrm{ab}$ \\
\hline Neutral & $0.54 \pm 0.02^{\mathrm{a}}$ & $0.55 \pm 0.03^{\mathrm{a}}$ & $0.53 \pm 0.03^{\mathrm{a}}$ & $0.59 \pm 0.03 \mathrm{bc}$ & $0.45 \pm 0.03^{\mathrm{ab}}$ \\
\hline Agree & $0.94 \pm 0.02^{b}$ & $0.91 \pm 0.03^{b}$ & $0.97 \pm 0.03^{b}$ & $1.01 \pm 0.02^{\mathrm{d}}$ & $0.72 \pm 0.03^{c}$ \\
\hline$p^{2}$ & $<0.001$ & \multicolumn{2}{|c|}{$<0.001$} & \multicolumn{2}{|r|}{$<0.001$} \\
\hline \multicolumn{6}{|c|}{ Friends' habit of eating fruit frequently } \\
\hline Disagree & $0.67 \pm 0.03^{\mathrm{a}}$ & $0.63 \pm 0.04^{\mathrm{a}}$ & $0.71 \pm 0.05^{\mathrm{ab}}$ & $0.75 \pm 0.04^{\mathrm{a}}$ & $0.49 \pm 0.03^{b d}$ \\
\hline Neutral & $0.67 \pm 0.02^{\mathrm{a}}$ & $0.68 \pm 0.03^{\mathrm{a}}$ & $0.67 \pm 0.03^{\mathrm{a}}$ & $0.73 \pm 0.03^{\mathrm{a}}$ & $0.56 \pm 0.03 \mathrm{bd}$ \\
\hline Agree & $0.93 \pm 0.03^{b}$ & $0.89 \pm 0.04^{b c}$ & $0.96 \pm 0.04^{\mathrm{c}}$ & $1.00 \pm 0.03^{c}$ & $0.69 \pm 0.04 \mathrm{ad}$ \\
\hline$p^{2}$ & $<0.001$ & \multicolumn{2}{|c|}{$<0.001$} & \multicolumn{2}{|r|}{$<0.001$} \\
\hline
\end{tabular}

${ }^{1}$ Values are fruit intake per day, presenting as means \pm standard errors. ${ }^{2} p$-values for differences between sexes or grade levels, obtained by ANOVA; letters $(\mathrm{a}, \mathrm{b}, \mathrm{c}, \mathrm{d}, \mathrm{e})$ indicate significant differences between groups (Scheffe's post-hoc test, $p<0.05$ ).

Table 4 shows the relationship between aspects of participants' food environments, frequency of fruit intakes, and weight status. The fruit intake frequency was not related to weight status in total subjects but as classified by grade; the lower intake frequency was shown in overweight groups of elementary school students. In terms of fruit environments and overweight, regular provision of fruit in school lunches and having a person at home to prepare fruit were negatively correlated to an overweight status for all participants. A low frequency of fruit intakes was associated with an overweight status among elementary school students. The proportion of overweight students was lower among those who perceived their food environment at school positively compared to those who did not $(p=0.044)$, especially for girls $(p=0.047)$ and elementary school students $(p=0.005)$. In addition, the proportion of overweight students was lower among those who had someone at home to prepare fruit compared to those who did not $(p<0.001)$, notably again for girls $(p=0.007)$ and elementary school students $(p=0.001)$. 
Table 4. Relationship between aspects of participants' food environments, frequency of fruit intakes, and weight status ${ }^{1}$.

\begin{tabular}{|c|c|c|c|c|c|c|c|c|c|c|}
\hline & \multirow{2}{*}{\multicolumn{2}{|c|}{ All }} & \multicolumn{4}{|c|}{ Sex } & \multicolumn{4}{|c|}{ Grade } \\
\hline & & & \multicolumn{2}{|c|}{ Boys } & \multicolumn{2}{|c|}{ Girls } & \multicolumn{2}{|c|}{ Elementary School } & \multicolumn{2}{|c|}{ Middle and High School } \\
\hline & $\begin{array}{l}\text { Normal } \\
\text { Weight }\end{array}$ & $\begin{array}{c}\text { Over } \\
\text { Weight }\end{array}$ & $\begin{array}{l}\text { Normal } \\
\text { Weight }\end{array}$ & $\begin{array}{c}\text { Over } \\
\text { Weight }\end{array}$ & $\begin{array}{l}\text { Normal } \\
\text { Weight }\end{array}$ & $\begin{array}{c}\text { Over } \\
\text { Weight }\end{array}$ & $\begin{array}{l}\text { Normal } \\
\text { Weight }\end{array}$ & $\begin{array}{c}\text { Over } \\
\text { Weight }\end{array}$ & $\begin{array}{l}\text { Normal } \\
\text { Weight }\end{array}$ & $\begin{array}{c}\text { Over } \\
\text { Weight }\end{array}$ \\
\hline \multicolumn{11}{|c|}{ Fruits intakes per day } \\
\hline$<0.5$ & 1194 (49.4) & $378(51.9)$ & $610(51.7)$ & $191(52.6)$ & $584(47.1)$ & $187(51.1)$ & $771(45.2)$ & $274(50.2)$ & $423(59.2)$ & $104(56.8)$ \\
\hline$\geq 0.5$ & $1225(50.6)$ & $351(48.1)$ & $569(48.3)$ & $172(47.4)$ & $656(52.9)$ & $179(48.9)$ & $934(54.8)$ & $272(49.8)$ & $291(40.8)$ & $79(43.2)$ \\
\hline$p^{2}$ & \multicolumn{2}{|c|}{0.238} & \multicolumn{2}{|c|}{0.770} & \multicolumn{2}{|c|}{0.179} & \multicolumn{2}{|c|}{0.043} & \multicolumn{2}{|c|}{0.554} \\
\hline \multicolumn{11}{|c|}{ Food environments } \\
\hline \multicolumn{11}{|c|}{ Availability of fruit at home } \\
\hline Disagree & $348(14.4)$ & $110(15.1)$ & $181(15.4)$ & $57(15.7)$ & $167(13.5)$ & $53(14.5)$ & $232(13.6)$ & $81(14.8)$ & $116(16.2)$ & $29(15.8)$ \\
\hline Neutral & $629(26.0)$ & $187(25.7)$ & $335(28.4)$ & $106(29.2)$ & $294(23.7)$ & $81(22.1)$ & $420(24.6)$ & $137(25.1)$ & $209(29.3)$ & $50(27.3)$ \\
\hline Agree & $1442(59.6)$ & $432(59.3)$ & $663(56.2)$ & $200(55.1)$ & $779(62.8)$ & $232(63.4)$ & $1053(61.8)$ & $328(60.1)$ & $389(54.5)$ & $104(56.8)$ \\
\hline$p^{2}$ & \multicolumn{2}{|c|}{0.892} & \multicolumn{2}{|c|}{0.929} & \multicolumn{2}{|c|}{0.768} & \multicolumn{2}{|c|}{0.713} & \multicolumn{2}{|c|}{0.838} \\
\hline \multicolumn{11}{|c|}{ Regular provision of fruit in school lunches } \\
\hline Disagree & $187(7.7)$ & $72(9.9)$ & $115(9.8)$ & $40(11.0)$ & $72(5.8)$ & $32(8.7)$ & $99(5.8)$ & $46(8.4)$ & $88(12.3)$ & $26(14.2)$ \\
\hline Neutral & $723(29.9)$ & $236(32.4)$ & $380(32.2)$ & $124(34.2)$ & $343(27.7)$ & $112(30.6)$ & $454(26.6)$ & $170(31.1)$ & $269(37.7)$ & $66(36.1)$ \\
\hline Agree & $1509(62.4)$ & $421(57.8)$ & $684(58.0)$ & $199(54.8)$ & $825(66.5)$ & $222(60.7)$ & $1152(67.6)$ & $330(60.4)$ & $357(50.0)$ & $91(49.7)$ \\
\hline$p^{2}$ & \multicolumn{2}{|c|}{0.044} & \multicolumn{2}{|c|}{0.535} & \multicolumn{2}{|c|}{0.047} & \multicolumn{2}{|c|}{0.005} & \multicolumn{2}{|c|}{0.775} \\
\hline \multicolumn{11}{|c|}{ Having someone at home to prepare fruit } \\
\hline Disagree & $310(12.8)$ & $129(17.7)$ & $160(13.6)$ & $66(18.2)$ & $150(12.1)$ & $63(17.2)$ & $204(12.0)$ & $91(16.7)$ & $106(14.8)$ & $38(20.8)$ \\
\hline Neutral & $539(22.3)$ & $179(24.6)$ & $297(25.2)$ & $96(26.4)$ & $242(19.5)$ & $83(22.7)$ & $342(20.1)$ & $129(23.6)$ & $197(27.6)$ & $50(27.3)$ \\
\hline Agree & $1570(64.9)$ & $421(57.8)$ & $722(61.2)$ & $201(55.4)$ & $848(68.4)$ & $220(60.1)$ & $1159(68.0)$ & $326(59.7)$ & $411(57.6)$ & $95(51.9)$ \\
\hline$p^{2}$ & & & & & & & & & & \\
\hline & & & & Family & bit of eating & it frequently & & & & \\
\hline Disagree & $211(8.7)$ & $71(9.7)$ & $107(9.1)$ & $36(9.9)$ & $104(8.4)$ & $35(9.6)$ & $141(8.3)$ & $52(9.5)$ & $70(9.8)$ & $19(10.4)$ \\
\hline Neutral & $730(30.2)$ & $224(30.7)$ & $393(33.3)$ & $125(34.4)$ & 337 (27.2) & $99(27.0)$ & $454(26.6)$ & $164(30.0)$ & $276(38.7)$ & $60(32.8)$ \\
\hline Agree & $1478(61.1)$ & $434(59.5)$ & $679(57.6)$ & $202(55.6)$ & $799(64.4)$ & $232(63.4)$ & $1110(65.1)$ & $330(60.4)$ & $368(51.5)$ & $104(56.8)$ \\
\hline$p^{2}$ & & & & & & & & & & \\
\hline & & & & Friend & bit of eating & it frequentl & & & & \\
\hline Disagree & $421(17.4)$ & $123(16.9)$ & $233(19.8)$ & $66(18.2)$ & $188(15.2)$ & 57 (15.6) & 275 (16.1) & 89 (16.3) & $146(20.4)$ & 34 (18.6) \\
\hline Neutral & 1051 (43.4) & $327(44.9)$ & $541(45.9)$ & $169(46.6)$ & $510(41.1)$ & $158(43.2)$ & $697(40.9)$ & $239(43.8)$ & $354(49.6)$ & 88 (48.1) \\
\hline Agree & $947(39.1)$ & 279 (38.3) & $405(34.4)$ & $128(35.3)$ & $542(43.7)$ & 151 (41.3) & $733(43.0)$ & $218(39.9)$ & $214(30.0)$ & $61(33.3)$ \\
\hline$p^{2}$ & & & & & & & & & & \\
\hline
\end{tabular}

${ }^{1}$ Values are presented as absolute numbers and percentages. ${ }^{2} p$-values for differences between sexes and grade levels, obtained by a chi-square test. 
Table 5. Relationship between aspects of participants' food environments and odds of being overweight.

\begin{tabular}{|c|c|c|c|c|c|c|c|c|}
\hline & \multicolumn{4}{|c|}{ Boys } & \multicolumn{4}{|c|}{ Girls } \\
\hline & \multicolumn{2}{|c|}{ Elementary School } & \multicolumn{2}{|c|}{ Middle and High School } & \multicolumn{2}{|c|}{ Elementary School } & \multicolumn{2}{|c|}{ Middle and High School } \\
\hline & $\mathrm{OR}^{1}(95 \% \mathrm{CI})$ & $\begin{array}{c}\text { Adjusted OR }{ }^{2} \\
\text { (A95\% CI) }\end{array}$ & OR $(95 \%$ CI $)$ & $\begin{array}{c}\text { Adjusted OR } \\
\text { (A95\% CI) }\end{array}$ & OR $(95 \%$ CI $)$ & $\begin{array}{l}\text { Adjusted OR } \\
\text { (A95\% CI) }\end{array}$ & OR $(95 \%$ CI $)$ & $\begin{array}{c}\text { Adjusted OR } \\
\text { (A95\% CI) }\end{array}$ \\
\hline \multicolumn{9}{|c|}{ Availability of fruit at home } \\
\hline Disagree & 1.00 & 1.00 & 1.00 & 1.00 & 1.00 & 1.00 & 1.00 & 1.00 \\
\hline Neutral & $0.87(0.56-1.35)$ & $0.87(0.56-1.34)$ & $1.41(0.69-2.90)$ & $1.41(0.69-2.88)$ & $1(0.63-1.60)$ & $1.02(0.64-1.62)$ & $0.61(0.29-1.29)$ & $0.61(0.29-1.27)$ \\
\hline Agree & $0.8(0.54-1.19)$ & $0.79(0.53-1.18)$ & $1.44(0.74-2.80)$ & $1.41(0.72-2.77)$ & $1(0.67-1.50)$ & $1.08(0.72-1.63)$ & $0.77(0.40-1.48)$ & $0.7(0.36-1.37)$ \\
\hline \multicolumn{9}{|c|}{ Regular provision of fruit in school lunches } \\
\hline Disagree & 1.00 & 1.00 & 1.00 & 1.00 & 1.00 & 1.00 & 1.00 & 1.00 \\
\hline Neutral & $0.87(0.52-1.47)$ & $0.87(0.52-1.47)$ & $0.98(0.49-1.96)$ & $1(0.50-1.99)$ & $0.72(0.39-1.30)$ & $0.72(0.40-1.31)$ & $0.66(0.30-1.44)$ & $0.71(0.32-1.55)$ \\
\hline Agree & $0.71(0.43-1.16)$ & $0.7(0.43-1.15)$ & $1.01(0.52-1.98)$ & $1.01(0.52-1.98)$ & $0.52(0.30-0.92)$ & $0.54(0.31-0.95)$ & $0.7(0.34-1.46)$ & $0.71(0.34-1.49)$ \\
\hline p-trend ${ }^{3}$ & 0.081 & 0.075 & 0.939 & 0.963 & 0.005 & 0.01 & 0.527 & 0.492 \\
\hline \multicolumn{9}{|c|}{ Having someone at home to prepare fruit } \\
\hline Disagree & 1.00 & 1.00 & 1.00 & 1.00 & 1.00 & 1.00 & 1.00 & 1.00 \\
\hline Neutral & $0.76(0.49-1.19)$ & $0.76(0.49-1.19)$ & $0.85(0.44-1.64)$ & $0.85(0.45-1.64)$ & $0.95(0.60-1.50)$ & $0.93(0.59-1.47)$ & $0.56(0.27-1.16)$ & $0.55(0.27-1.14)$ \\
\hline p-trend ${ }^{3}$ & 0.023 & 0.019 & 0.275 & 0.223 & 0.004 & 0.009 & 0.122 & 0.06 \\
\hline \multicolumn{9}{|c|}{ Family habit of eating fruit frequently } \\
\hline Disagree & 1.00 & 1.00 & 1.00 & 1.00 & 1.00 & 1.00 & 1.00 & 1.00 \\
\hline Neutral & $1.16(0.69-1.93)$ & $1.15(0.68-1.92)$ & $0.63(0.29-1.36)$ & $0.63(0.29-1.36)$ & $0.81(0.48-1.37)$ & $0.82(0.49-1.38)$ & $1.08(0.45-2.58)$ & $1.03(0.43-2.46)$ \\
\hline Agree & $0.83(0.51-1.36)$ & $0.81(0.49-1.33)$ & $1.01(0.48-2.12)$ & $1.00(0.47-2.13)$ & $0.78(0.49-1.25)$ & $0.84(0.52-1.36)$ & $1.09(0.47-2.50)$ & $0.96(0.41-2.25)$ \\
\hline p-trend ${ }^{3}$ & 0.097 & 0.081 & 0.301 & 0.346 & 0.357 & 0.649 & 0.879 & 0.835 \\
\hline \multicolumn{9}{|c|}{ Friends' habit of eating fruit frequently } \\
\hline Disagree & 1.00 & 1.00 & 1.00 & 1.00 & 1.00 & 1.00 & 1.00 & 1.00 \\
\hline Neutral & $1.06(0.72-1.56)$ & $1.06(0.72-1.56)$ & $1.26(0.69-2.31)$ & $1.25(0.69-2.29)$ & $1.06(0.70-1.59)$ & $1.04(0.69-1.58)$ & $0.86(0.45-1.65)$ & $0.85(0.44-1.63)$ \\
\hline Agree & $1.02(0.69-1.52)$ & $1.02(0.69-1.51)$ & $1.31(0.67-2.57)$ & $1.29(0.65-2.54)$ & $0.84(0.56-1.27)$ & $0.86(0.57-1.31)$ & $1.13(0.59-2.17)$ & $1.07(0.55-2.08)$ \\
\hline$p$-trend ${ }^{3}$ & 0.962 & 0.977 & 0.459 & 0.496 & 0.204 & 0.302 & 0.586 & 0.699 \\
\hline
\end{tabular}

(ORs) were derived from logistic regression models. ${ }^{2}$ Adjusted ORs adjusted for fruit intake frequency and were derived from logistic regression models. ${ }^{3} p$-values for trends. 
Table 5 shows the relationship between aspects of participants' food environments and odds of being overweight, unadjusted and adjusted for fruit intake frequency. For boys, those who had someone at home to prepare fruit were unlikely to be overweight $(\mathrm{OR}=0.64,95 \% \mathrm{CI}=0.43-0.94$, p-trend $=0.023)$, and these results remained significant after adjusting for frequency of fruit intakes $(\mathrm{OR}=0.63,95 \% \mathrm{CI}=0.420 .93, p$-trend $=0.019)$. For elementary school girls, regular provision of fruit in school lunches $(\mathrm{OR}=0.52,95 \% \mathrm{CI}=0.30-0.92, p$-trend $=0.005)$ and having someone at home to prepare fruit for them $(\mathrm{OR}=0.63,95 \% \mathrm{CI}=0.43-0.93, p$-trend $=0.004)$ were negatively related to an overweight status, even after adjusting for frequency of fruit intakes $(\mathrm{OR}=0.54,95 \% \mathrm{CI}=0.31-0.95, p$-trend $=0.01$ for regular provision of fruit in school lunches; $\mathrm{OR}=0.65,95 \% \mathrm{CI}=0.44-0.96, p$-trend $=0.009$ for having someone at home to prepare fruit). Among middle and high school girls, having someone at home to prepare fruit was significantly associated with a lower risk of being overweight after adjusting for frequency of fruit intakes ( $\mathrm{OR}=0.51,95 \% \mathrm{CI}=0.27-0.96$, $p$-trend $=0.06)$.

\section{Discussion}

Because of the prominent influence that food environments exert on an individual's tendency toward obesity, we explored the relationship between aspects of the home and school food environments, fruit intakes, and overweight status among disadvantaged children and adolescents. We found that the frequency of fruit intakes increased when participants perceived their food environments positively, and this was associated with a reduced prevalence of overweight. In particular, regular provision of fruit in school lunches and having someone at home to prepare fruit was associated with a healthier body weight among elementary school students and girls.

Fruit was consumed an average of 0.77 times per day by our participants, which was below the recommended level of the Dietary Reference Intake for Koreans of at least twice per day [31]. According to the Korea National Health and Nutrition Examination Survey [32], low-income families consume less fruit than medium-income families (100.1 vs. $135.2 \mathrm{~g} /$ day). In this study, frequency of fruit intake varied according to a participant's perception of their food environment, with higher rates of consumption among those who responded positively. These results provide evidence that food environments to increase fruit intakes would play an important role in fruit consumption.

Previously, review papers and meta-analyses have shown that increasing the availability and accessibility of fruits and vegetables for children at school is effective at preventing obesity [33,34]. In this study, regular provision of fruit in school lunches was positively related to fruit intakes among elementary school students, but not middle and high school students. These findings do not align with those from a related investigation in the United States, wherein high school students who received fruits and vegetables from The Fresh Fruit and Vegetable Program were likely to eat fruit more often than those who did not (59.1\% vs. $40.9 \%$ ) [35]. Given that middle and high school students appear to have more established eating habits, it may be necessary to provide fruit at a higher frequency in order to enhance intakes $[35,36]$. The school lunch service in Korea could provide fruits as a dessert within the school budget, but the current frequency of provision of fruit at school lunch would not be enough to meet the consumption of fruit for the disadvantaged middle and high school students in Korea. Therefore, additional funding for more frequent provision of fruit at school lunch should be needed to increase their fruit consumption.

Regarding the food environment at home, having someone to prepare fruit for students was found to relate favorably to fruit intakes and weight status. This result was consistent with those from other studies reporting that social support for healthy food intakes aides in the prevention of obesity among vulnerable children $[37,38]$. A few reasons might explain this. First, caregivers give children fruit in a form that allows them to eat it easily. Several studies have shown that providing fruit in a ready-to-eat form or making it visible to children promotes fruit intakes $[39,40]$. Because the process of washing, cutting, and peeling fruit has been described as an impediment to consumption, providing or storing it in an accessible form may help children eat fruit more frequently [40,41]. Indeed, analyses from the Healthy Habits randomized trial [42] revealed that the frequency of fruit provision from a parent 
positively impacted their children's fruit intakes after 12 months. Second, caregivers could promote fruit intakes simply by encouraging their children [43]. Considering that participants in our study hailed from disadvantaged backgrounds, it is unlikely that their caregivers purchase much fruit for them or monitor their intakes at home. Thus, incorporating the Healthy Fruit Basket Program into all Community Childcare Centers and related institutions nationwide may help these individuals increase fruit intakes and attenuate risk of overweight or obesity $[25,26]$.

It is well established that availability of food in the home is a key determinant of consumption among children and adolescents [44-46], and our analyses confirmed this phenomenon for fruit, in particular. Additionally, we observed that a family's habit of eating fruit frequently was connected to high fruit intakes across all participants, similar to findings from other publications $[39,42,43]$.

Interestingly, this investigation detected no difference in fruit intakes between sexes sharing the same perception about their food environments. However, with respect to the grade levels, elementary school students were found to consume fruit more frequently than middle or high school students when their food environment was perceived positively. Childhood is a period of social modeling, as individuals learn how to interact with their environments and behave appropriately $[11,47,48]$. Hence, the benefits of food environments on fruit intakes may apply more strongly to children than adolescents. For another explanation, a recent study of adults with low socioeconomic position found that self-efficacy on fruit and vegetable consumption was more strongly associated with fruit and vegetable consumption than perceived food environments, which implies the importance of capacity building to partially overcome the poor food environment [49]. As several studies have shown the positive effect of nutritional education on fruit and vegetable intakes [50-52], nutritional education intervention should be included for adolescents with negative perception of food environment who especially have low self-efficacy to increase their fruit and vegetable intakes.

When we examined the relationship between fruit intakes and weight status, a high frequency ( $>0.5$ times per day) was tied to a lower prevalence of overweight in girls. According to systematic reviews, consumption of fruit as a means to prevent pediatric obesity did not always produce consistent results in terms of gender or amount $(38,39)$. For example, one investigation noted that eating fruit more than twice a day was only protective against obesity in boys [53], and another showed that excessive fruit intakes actually engendered obesity [54]. Heeding our results and those from earlier studies, consumption of fruit alone may not be adequate to prevent obesity. Specifically, our investigation found that the effects of some aspects of the food environment on the prevalence of obesity remained significant after adjusting for fruit intake frequency, and a related study observed a positive link between parental concern for their children's diet and fitness practices $(r=0.552, p<0.001)$ [55]. It could suggest that the home environment supporting children to eat fruits could also support other behaviors that can prevent obesity in children, such as encouraging exercise. Further research exploring caretakers' interest in their children's health will provide a more holistic understanding of how environments moderate their risk of obesity.

The present study had several limitations and strengths. Concerning limitations, this was a cross-sectional study, so any causal associations between food environments, fruit intakes, and overweight status have yet to be determined. In addition, the questionnaire evaluating fruit availability and accessibility in the home and school food environments was not validated, although it had been employed in a previous study [30]. Moreover, we did not collect information pertaining to other confounders of overweight, which could offer deeper insight into factors underlying the present findings $[25,26]$. Granted, this was a highly homogenous cohort since all participants were recruited from the Community Childcare Center, so it is unlikely they possessed any remarkable characteristics that would alter our results. Nonetheless, future studies examining a more diverse population are needed to confirm the relationship between the food environment and risk of obesity by adjusting the confounding factor. Despite limitations, these results provide evidence that food environments play an important role in overweight prevention as well as fruit consumption among underprivileged Asian children and adolescents, using a relatively large sample size. 


\section{Conclusions}

This study found that the frequency of fruit intakes was generally low among children and adolescents from disadvantaged backgrounds; however, those who perceived their food environments positively consumed more fruit and were less likely to be overweight than those who perceived them negatively. Noteworthily, regular provision of fruit in school lunches and having someone at home to prepare fruit were predictive of a high frequency of fruit intakes and lower prevalence of overweight, and these discoveries were most apparent among elementary school students and girls. On the whole, our findings demonstrate that augmenting access to fruit within any realm of the food environment is associated with increased consumption and healthier body weights for low-income youth. Going forward, the school lunch service in Korea could consider increasing fruit servings since current provisions are not sufficient for enabling disadvantaged middle and high school students to meet the dietary guidelines. In addition, a nation-wide program to increase fruit consumption at the Community Childcare Center where they usually spend time would be essential to improve their health.

Author Contributions: K.K. conceived and designed the study; N.Y. analyzed the data and wrote the first draft of the paper; K.K. supervised the data analysis and revised the draft paper. All authors have read and agreed to the published version of the manuscript.

Funding: This research was funded by the National Research Foundation (NRF) of Korea (2019R1A2C1084372).

Conflicts of Interest: All authors declare no conflict of interest.

\section{References}

1. Di Cesare, M.; Sorić, M.; Bovet, P.; Miranda, J.J.; Bhutta, Z.; Stevens, G.A.; Laxmaiah, A.; Kengne, A.P.; Bentham, J. The epidemiological burden of obesity in childhood: A worldwide epidemic requiring urgent action. BMC Med. 2019, 17, 1-20. [CrossRef]

2. Rankin, J.; Matthews, L.; Cobley, S.; Han, A.; Sanders, R.; Wiltshire, H.D.; Baker, J.S. Psychological consequences of childhood obesity: Psychiatric comorbidity and prevention. Adolesc. Health Med. Ther. 2016, 7, 125-146. [CrossRef]

3. Sagar, R.; Gupta, T. Psychological Aspects of Obesity in Children and Adolescents. Indian J. Pediatr. 2018, 85, 554-559. [CrossRef]

4. Anthropometric Measures during Infancy and Childhood and the Risk of Developing Cardiovascular Disease or Diabetes Mellitus Type 2 in Later Life: A Systematic Review 2015. Available online: https://www.who.int/ maternal_child_adolescent/documents/singh-anthropometry-ncd-infants-children-2015.pdf?ua=1 (accessed on 27 August 2020).

5. Preventing and Managing the Global Epidemic-WHO Technical Report Series. Available online: https: //www.who.int/nutrition/publications/obesity/WHO_TRS_894/en/ (accessed on 27 August 2020).

6. Dehghan, M.; Akhtar-Danesh, N.; Merchant, A.T. Childhood obesity, prevalence and prevention. Nutr. J. 2005, 4, 1-8. [CrossRef]

7. Bentham, J.; Di Cesare, M.; Bilano, V.; Bixby, H.; Zhou, B.; Stevens, G.A.; Riley, L.M.; Taddei, C.; Hajifathalian, K.; Lu, Y.; et al. Worldwide trends in body-mass index, underweight, overweight, and obesity from 1975 to 2016: A pooled analysis of 2416 population-based measurement studies in 128.9 million children, adolescents, and adults. Lancet 2017, 390, 2627-2642. [CrossRef]

8. Poti, J.; Braga, B.; Quin, B. Ultra-processed food intake and obesity: What really matters for health—Processing or nutrient content? Curr. Obes. Rep. 2018, 6, 420-431. [CrossRef]

9. Huang, J.-Y.; Qi, S.-J. Childhood obesity and food intake. World J. Pediatr. 2015, 11, 101-107. [CrossRef]

10. Epstein, L.H.; Paluch, R.A.; Beecher, M.D.; Roemmich, J.N. Increasing healthy eating vs. reducing high energy-dense foods to treat pediatric obesity. Obesity 2008, 16, 318-326. [CrossRef]

11. Ricotti, R.; Caputo, M.; Prodam, F. Chapter 9-Mediterranean diet, nutrition transition, and cardiovascular risk factor in children and adolescents. In The Mediterranean Diet: An Evidence-Based Approach, 2nd ed.; Preedy, V.R., Watson, R.R., Eds.; Academic Press: Cambridge, MA, USA, 2020; pp. 89-95, ISBN 978-0-12-818649-7. 
12. Rolls, B.J.; Ello-Martin, J.A.; Tohill, B.C. What Can Intervention Studies Tell Us about the Relationship between Fruit and Vegetable Consumption and Weight Management? Nutr. Rev. 2004, 62, 1-17. [CrossRef]

13. Aune, D.; Giovannucci, E.; Boffetta, P.; Fadnes, L.T.; Keum, N.N.; Norat, T.; Greenwood, D.C.; Riboli, E.; Vatten, L.J.; Tonstad, S. Fruit and vegetable intake and the risk of cardiovascular disease, total cancer and all-cause mortality-A systematic review and dose-response meta-analysis of prospective studies. Int. J. Epidemiol. 2017, 46, 1029-1056. [CrossRef]

14. Hebden, L.; O’Leary, F.; Rangan, A.; Singgih Lie, E.; Hirani, V.; Allman-Farinelli, M. Fruit consumption and adiposity status in adults: A systematic review of current evidence. Crit. Rev. Food Sci. Nutr. 2017, 57, 2526-2540. [CrossRef]

15. Stamler, J.; Dolecek, T. Chapter 13. Relation of food and nutrient intakes to body mass in the special intervention and usual care groups in the Multiple Risk Factor Intervention Trial. Am. J. Clin. Nutr. 1997, 65, 366S-373S. [CrossRef]

16. Buijsse, B.; Feskens, E.J.M.; Schulze, M.B.; Forouhi, N.G.; Wareham, N.J.; Sharp, S.; Palli, D.; Tognon, G.; Halkjaer, J.; Tjønneland, A.; et al. Fruit and vegetable intakes and subsequent changes in body weight in European populations: Results from the project on Diet, Obesity, and Genes (DiOGenes). Am. J. Clin. Nutr. 2009, 90, 202-209. [CrossRef]

17. Kamath, C.C.; Vickers, K.S.; Ehrlich, A.; McGovern, L.; Johnson, J.; Singhal, V.; Paulo, R.; Hettinger, A.; Erwin, P.J.; Montori, V.M. Behavioral interventions to prevent childhood obesity: A systematic review and metaanalyses of randomized trials. J. Clin. Endocrinol. Metab. 2008, 93, 4606-4615. [CrossRef]

18. Swinburn, B.; Egger, G.; Raza, F. The Development and Application of a Framework for Identifying and Prioritizing Environmental Interventions for Obesity. Dissecting Obesog. Environ. 1999, 29, 563-570.

19. Rideout, K.; Mah, C.; Minaker, L. Food Environments: An Introduction for Public Health Practice; National Collaborating Centre for Environmental Health British Columbia Centre for Disease Control: Vancouver, BC, Canada, 2015.

20. Rosenkranz, R.R.; Dzewaltowski, D.A. Model of the home food environment pertaining to childhood obesity. Nutr. Rev. 2008, 66, 123-140. [CrossRef]

21. Chan, K.; Prendergast, G.; Grønhøj, A.; Bech-Larsen, T. The role of socializing agents in communicating healthy eating to adolescents: A cross-cultural study. J. Int. Consum. Mark. 2011, 23, 59-74. [CrossRef]

22. Food and Health in Europe: A New Basis for Action. Available online: https://www.who.int/nutrition/ publications/Food_and_health_Europe\%20_newbasis_for_\%20action.pdf (accessed on 27 August 2020).

23. Lytle, L.A. Measuring the Food Environment. State of the Science. Am. J. Prev. Med. 2009, 36, 1-18. [CrossRef]

24. Micha, R.; Karageorgou, D.; Bakogianni, I.; Trichia, E.; Whitsel, L.P.; Story, M.; Peñalvo, J.L.; Mozaffarian, D. Effectiveness of school food environment policies on children's dietary behaviors: A systematic review and meta-analysis. PLoS ONE 2018, 13, 1-27. [CrossRef]

25. Ministry of Health and Welfare. 2015 Community Child Centers Support Program Manual; Ministry of Health and Welfare: Sejong, Korea, 2015; p. 19.

26. Ministry of Health and Welfare; Korea Health Promotion Institute. Healthy Fruit Basket Program Manual; Korea Health Promotion Institute: Sejong, Seoul, 2013; p. 9.

27. Aarestrup, J.; Gamborg, M.; Ulrich, L.G.; Sørensen, T.I.A.; Baker, J.L. Childhood body mass index and height and risk of histologic subtypes of endometrial cancer. Int. J. Obes. 2016, 40, 1096-1102. [CrossRef]

28. Nogueira, L.; Stolzenberg-Solomon, R.; Gamborg, M.; Sørensen, T.I.; Baker, J.L. Childhood Body Mass Index and Risk of Adult Pancreatic Cancer. Curr. Dev. Nutr. 2017, 1, e001362. [CrossRef]

29. Korea Centers for Disease Control and Prevention; Committee for the Development of Growth Standard for Korean Children and Adolescents; Korean Pediatric Society. 2007 Korean Children and Adolescents Growth Standard (Commentary for the Development of 2007 Growth Chart); Korea Centers for Disease Control and Prevention: Cheongju, Korea, 2007; pp. 93-104.

30. Story, M.; Kaphingst, K.M.; Robinson-O’Brien, R.; Glanz, K. Creating Healthy Food and Eating Environments: Policy and Environmental Approaches. Annu. Rev. Public Health 2008, 29, 253-272. [CrossRef]

31. The Ministry of Health and Welfare; The Korean Nutrition Society. Dietary Reference Intakes for Koreans 2015; The Korean Nutrition Society: Sejong, Korea, 2015; pp. 958-959. 
32. The Ministry of Health and Welfare; Korea Centers for Disease Control and Prevention. Korea Health Statistics 2018: Korea National Health and Nutrition Examination Survey (KNHANES VII-3); Korea Centers for Disease Control and Prevention: Sejong, Korea, 2020.

33. Pineda, E.; Swinburn, B.; Sassi, F. Effective school food environment interventions for the prevention of childhood obesity: Systematic review and meta-analysis. Lancet 2019, 394, S77. [CrossRef]

34. Bere, E.; Klepp, K.I.; Øverby, N.C. Free school fruit: Can an extra piece of fruit every school day contribute to the prevention of future weight gain? A cluster randomized trial. Food Nutr. Res. 2014, 58, 1-5. [CrossRef]

35. Davis, E.M.; Cullen, K.W.; Watson, K.B.; Konarik, M.; Radcliffe, J. A Fresh Fruit and Vegetable Program Improves High School Students' Consumption of Fresh Produce. J. Am. Diet. Assoc. 2009, 109, 1227-1231. [CrossRef]

36. Ridberg, R.A.; Bell, J.F.; Merritt, K.E.; Harris, D.M.; Young, H.M.; Tancredi, D.J. Effect of a fruit and vegetable prescription program on children's fruit and vegetable consumption. Prev. Chronic Dis. 2019, 16, 1-13. [CrossRef]

37. Story, M. School-based approaches for preventing and treating obesity. Int. J. Obes. 1999, 23, S43-S51. [CrossRef]

38. Heredia, N.I.; Ranjit, N.; Warren, J.L.; Evans, A.E. Association of parental social support with energy balance-related behaviors in low-income and ethnically diverse children: A cross-sectional study. BMC Public Health 2016, 16, 1-12. [CrossRef]

39. Ray, C.; Roos, E.; Brug, J.; Behrendt, I.; Ehrenblad, B.; Yngve, A.; Te Velde, S.J. Role of free school lunch in the associations between family-environmental factors and children's fruit and vegetable intake in four European countries. Public Health Nutr. 2013, 16, 1109-1117. [CrossRef]

40. Wyse, R.; Campbell, E.; Nathan, N.; Wolfenden, L. Associations between characteristics of the home food environment and fruit and vegetable intake in preschool children: A cross-sectional study. BMC Public Health 2011, 11, 938. [CrossRef]

41. McMorrow, L.; Ludbrook, A.; Macdiarmid, J.I.; Olajide, D. Perceived barriers towards healthy eating and their association with fruit and vegetable consumption. J. Public Health 2017, 39, 330-338. [CrossRef]

42. Wyse, R.; Wolfenden, L.; Bisquera, A. Characteristics of the home food environment that mediate immediate and sustained increases in child fruit and vegetable consumption: Mediation analysis from the Healthy Habits cluster randomised controlled trial. Int. J. Behav. Nutr. Phys. Act. 2015, 12, 1-9. [CrossRef]

43. Pearson, N.; Biddle, S.J.H.; Gorely, T. Family correlates of fruit and vegetable consumption in children and adolescents: A systematic review. Public Health Nutr. 2009, 12, 267-283. [CrossRef]

44. Yee, A.Z.H.; Lwin, M.O.; Ho, S.S. The influence of parental practices on child promotive and preventive food consumption behaviors: A systematic review and meta-analysis. Int. J. Behav. Nutr. Phys. Act. 2017, 14, 1-14. [CrossRef]

45. Befort, C.; Kaur, H.; Nollen, N.; Sullivan, D.K.; Nazir, N.; Choi, W.S.; Hornberger, L.; Ahluwalia, J.S. Fruit, vegetable, and fat intake among non-hispanic black and non-hispanic white adolescents: Associations with home availability and food consumption settings. J. Am. Diet. Assoc. 2006, 106, 367-373. [CrossRef]

46. Spurrier, N.J.; Magarey, A.A.; Golley, R.; Curnow, F.; Sawyer, M.G. Relationships between the home environment and physical activity and dietary patterns of preschool children: A cross-sectional study. Int. J. Behav. Nutr. Phys. Act. 2008, 5, 1-12. [CrossRef]

47. Goslin, D.A. Handbook of Socialization Theory and Research, 4th ed.; Rand McNally: Chicago, IL, USA, 1969; pp. 213-262, ISBN 0528686801.

48. Schmitt, R. The Stages of Moral Development: A Basis for an Educational Concept? Int. Rev. Educ. 1980, 26, 207-216.

49. de Menezes, M.C.; Diez Roux, A.V.; Souza Lopes, A.C. Fruit and vegetable intake: Influence of perceived food environment and self-efficacy. Appetite 2018, 127, 249-256. [CrossRef]

50. Wagner, M.G.; Rhee, Y.; Honrath, K.; Blodgett Salafia, E.H.; Terbizan, D. Nutrition education effective in increasing fruit and vegetable consumption among overweight and obese adults. Appetite 2016, 100, 94-101. [CrossRef]

51. Honrath, K.; Wagner, M.G.; Rhee, Y. Does Nutrition Education with Fruit and Vegetable Supplementation Increase Fruit and Vegetable Intake and Improve Anthropometrics of Overweight or Obese People of Varying Socioeconomic Status? Ecol. Food Nutr. 2018, 57, 32-49. [CrossRef] 
52. Clark, R.L.; Famodu, O.A.; Holásková, I.; Infante, A.M.; Murray, P.J.; Olfert, I.M.; McFadden, J.W.; Downes, M.T.; Chantler, P.D.; Duespohl, M.W.; et al. Educational intervention improves fruit and vegetable intake in young adults with metabolic syndrome components. Nutr. Res. 2019, 62, 89-100. [CrossRef]

53. Santiago, S.; Zazpe, I.; Martí, A.; Cuervo, M.; Martínez, J.A. Gender differences in lifestyle determinants of overweight prevalence in a sample of Southern European children. Obes. Res. Clin. Pract. 2013, 7, e391-e400. [CrossRef]

54. Field, A.E.; Gillman, M.W.; Rosner, B.; Rockett, H.R.; Colditz, G.A. Association between fruit and vegetable intake and change in body mass index among a large sample of children and adolescents in the United States. Int. J. Obes. 2003, 27, 821-826. [CrossRef]

55. Attorp, A.; Scott, J.E.; Yew, A.C.; Rhodes, R.E.; Barr, S.I.; Naylor, P.J. Associations between socioeconomic, parental and home environment factors and fruit and vegetable consumption of children in grades five and six in British Columbia, Canada. BMC Public Health 2014, 14, 1-9. [CrossRef]

Publisher's Note: MDPI stays neutral with regard to jurisdictional claims in published maps and institutional affiliations.

(C) 2020 by the authors. Licensee MDPI, Basel, Switzerland. This article is an open access article distributed under the terms and conditions of the Creative Commons Attribution (CC BY) license (http://creativecommons.org/licenses/by/4.0/). 